\title{
Rational use of regional nodal irradiation needed
}

I: rradiation of the axillary, supraclavicular and/or internal mammary (regional) lymph nodes is part of adjuvant therapy for breast cancer. This approach has been examined over many decades, with the aim of combating common lymphnode metastases. Initial results were disappointing, and non-cancer-related mortality owing to cardiac irradiation was a valid concern. Advances in radiotherapy, however, resulted in improved radiation delivery and outcomes. As Philip Poortmans explains, "it was time to conduct trials of nodal irradiation, coupled to good qualityassurance programmes for the radiotherapy delivered." Two such trials, the EORTC 22922-10925 trial, led by Poortmans, and the National Cancer Institute of Canada MA.20 trial by Tim Whelan and colleagues, have now been published.

The EORTC and MA.20 trials randomly assigned women with early stage breast cancer ( $n=4,004$ and $n=1,832$, respectively) to receive radiotherapy to the whole breast (or chest wall after mastectomy for $\sim 24 \%$ of women in the EORTC trial) either with or without regional nodal irradiation. Both trials reported data after a median follow-up duration of $\sim 10$ years. The results of both studies were similar.

Poortmans summarizes the EORTC trial data: "nodal irradiation resulted in significantly improved disease-free survival; significantly less distant metastasis; significantly reduced breast-cancerrelated mortality; a borderline significant improvement in overall survival; limited toxicity; and no treatment-related mortality, including from cardiac adverse events." Tim Whelan adds, "the most significant findings are that patients treated with regional radiation plus whole-breast irradiation had a reduced risk of breast-cancer recurrence, not only in the regional nodes, but also at distant sites. This is important because disease that spreads distantly invariably leads to death." He continues, "at this point in follow-up, however, we still have not seen overall improvement in survival, but follow-up is still just less than 10 years."

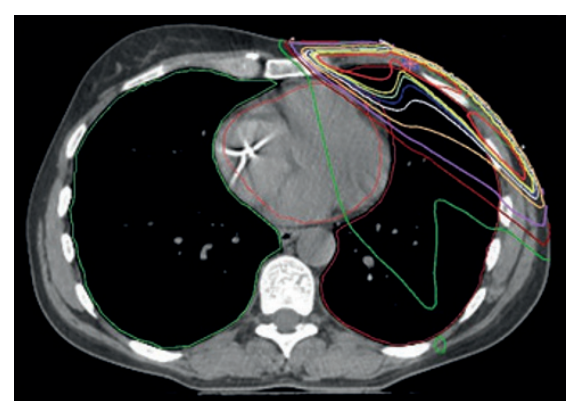

Axial CT image showing isodose distribution at the central slice, using an individualized 3D conformal radiotherapy technique without respiratory control for regional nodal irradiation, as used by some participating centres in the EORTC 22922-10925 trial. Image courtesy of Philip Poortmans.

Nevertheless, the lack of a statistically significant overall survival advantage, and the increased risks of lymphoedema, pneumonitis and cutaneous adverse events observed, mean that the debate over the use of regional nodal irradiation is likely to continue. Particularly considering, as Whelan highlights, "regional radiation is more complex, time-consuming, and more expensive to deliver." In fact, these studies highlight the persistent dilemmas relating to the use of nodal irradiation in women with early stage breast cancer, as outlined nicely by Burstein and Morrow in an editorial accompanying the trial publications.

Most patients were treated with adjuvant systemic therapies, but in the EORTC trial (as highlighted by Burstein and Morrow, and as acknowledged by the investigators), in an era (1996-2004) before the universal use of the highly effective contemporary regimens. This factor might be especially important in light of data suggesting that systemic therapy can negate the need for axillary dissection, without additional nodal irradiation, even in node-positive patients.

Furthermore, the clinical and molecular risk factors for locoregional and distant recurrence, including the importance of the extent of nodal involvement, were only beginning to be appreciated when these trials were initiated. Understanding of predictors of recurrence has advanced considerably since then, and the potential to personalize adjuvant treatment accordingly has grown. The value of nodal irradiation is unclear and the use of this treatment is most controversial in patients with $1-3$ positive nodes; around $43 \%$ and $85 \%$ of women in the EORTC and MA.20 trials, respectively, fell within this group, but assessments of this and other subgroups were limited.

Burstein and Morrow also suggested that the potential benefit of nodal irradiation might be dependent on the extent of axillary-lymph-node dissection achieved. In the MA.20 and EORTC trials, respectively, a median of 12 and 14 nodes were removed, $90 \%$ and $56 \%$ of patients had positive nodes, and the 10-year regional nodal recurrence rates among those who did not receive nodal irradiation were $2.7 \%$ and $4.7 \%$. By comparison, in trials of axillary-lymph-node dissection in women who all had at least one positive sentinel node, removal of a median of 17 nodes has been associated with recurrence rates of $<0.5 \%$ - admittedly, considering the axilla only and after only 5 years of follow-up.

These issues emphasize the need to rationally assign adjuvant therapies for breast cancer, including regional nodal irradiation and/or axillary dissection, according to the risk of recurrence, and the need for further study in this area. This fact is acknowledged by Poortmans: "We will do further subgroup analysis to define the patients who are most likely to benefit from regional nodal irradiation, and we intend to develop a shared decision-making tool for this purpose." Whelan adds, "we plan to do further research to identify potential biomarkers for identification of patients at high risk of recurrence and who are more likely to respond to nodal irradiation."

\section{David Killock}

